\title{
Conception et exécution des tunnels: rôle et résultats de la recherche expérimentale
}

\section{P. LUNARDI}

Rock Soil

Via Petrarca, 3

24100 Bergame (Italie)
Cet article résume les résultats d'une recherche expérimentale menée depuis vingt-cinq années, selon un programme qui a été appliqué systématiquement au cours de la construction de plus de $250 \mathrm{~km}$ de tunnels, en présence des types les plus divers de terrain.

soumis à différents états de contrainte.

Les résultats permettent de montrer comment

la rigidité du noyau de terrain au front de taille (noyau

d'avancement) influence le comportement contrainte-

déformation du tunnel et comment il est possible

d'utiliser le même noyau comme instrument de stabilisation.

\section{Tunnel design and construction: importance and results of experimental research}

This article illustrates the results of experimental research carried out by the author over the last 25 years. The research was based on a programme that was systematically implemented during the construction of more than $250 \mathrm{~km}$ of tunnel driven through many different types of ground subject to different stress-strain conditions.

The results show that the rigidity of the core of ground ahead of the face (the advance core) affects the stress-strain behaviour of a tunnel, and that it is possible to use this core as a tool to regulate the short and long term stability of a cavity.
} 


\section{Introduction}

On peut résumer comme suit les considérations développées dans un article précédent intitulé «Présoutènement et préconfinement s) [12] :

1) la tenue et la durée d'une excavation dans le sous-sol dépendent de la formation de l'effet de voûte, autrement dit de la déviation des contraintes sur le contour de la cavité:

2) par voie de conséquence, l'objectif premier et le plus important du projeteur de tunnels consiste à étudier si l'effet de voûte peut s'amorcer au moment de l'excavation d'une cavité et comment

3) la confirmation de la mobilisation de l'effet de voûte est fournie par la lecture et par l'interprétation de la réponse en déformation du milieu à l'action d'excavation:

4) la réponse en déformation se produit en amont du front de taille, dans le cadre de la zone perturbée à la suite de l'augmentation des contraintes provoquées dans le milieu sur le contour de l'excavation. Elle dépend de la nature du milieu soumis à un certain état de contrainte (consistance) et des modalités suivies dans la réalisation de l'avancement du front (action);

5) la réponse en déformation se manifeste tout d'abord au front de taille, par des phénomènes d'extrusion et de préconvergence, puis sur le contour de la cavité par des phénomènes de convergence du front conditionnés dans une large mesure par les premiers. Extrusion, préconvergence et convergence dépendent directement ou indirectement de la rigidité du noyau au front ou noyau d'avancement et donc de même que toutes les manifestations d'instabilité qui s'ensuivent:

6) dès lors, la stabilité d'un tunnel ne peut pas ne pas dépendre de celle de son noyau au front de taille, dont le comportement contrainte-déformation devra faire l'objet d'une étude prioritaire de la part du projeteur de tunnels

7) dans cette optique, le problème de la stabilité d'un tunnel est intrinsèquement tridimensionnel et sera traité en tant que tel même en ce qui concerne le choix des instruments de calcul;

8) le noyau d'avancement peut être vu comme l'instrument principal de la stabilisation à court et à long terme du tunnel. A court terme car il est à même de conditionner la réponse en déformation. A long terme car il est à même d'uniformiser et de réduire au maximum les charges qui se mobilisent, à la suite de l'avancement, sur le revêternent final et sur la base desquelles ce dernier est dimensionné et vérifié. Le comportement du noyau peut donc être adopté comme référence pour un nouveau type d'encadrement des ouvrages souterrains, et non par des classes géomécaniques mais sur des catégories de comportement contrainte-déformation:

9) cette découverte a permis, d'une part, de mettre au point le nouveau concept de préconfinement de la cavité ainsi que des interventions innovantes de stabilisation ( (cinterventions de conservation $)$ ) et, d'autre part, de commencer à penser à une nouvelle approche de conception et de construction, basée sur l'analyse des déformations contrôlées dans les roches et dans les sols, valable pour n'importe quel type de terrain et dans n'importe quelle condition de contrainte et de déformation (A.DE.CO-RS);

10) il est devenu enfin possible de concevoir et de construire des tunnels quel que soit le contexte géomécanique et quelle que soit la situation de contrainte et de déformation, tout en respectant les délais et les coûts prévus par le devis.

Nous sommes parvenus à ces importantes conclusions après vingt-cinq années de recherches menées selon un programme d'études et d'observations qui s'est développé progressivement en trois phases principales et qui a été appliqué systématiquement au cours de la construction de plus de $250 \mathrm{~km}$ de tunnels, en présence des types les plus divers de terrain, assujettis à différents états de contrainte.

A ce stade, avant d'entrer dans le détail et d'exposer les concepts de base de la nouvelle approche de conception et de construction des tunnels basée sur l'analyse des déformations contrôlées dans les roches et dans les sols, il est indispensable d'illustrer la recherche et les observations qui nous ont mené à formuler les affirmations précédentes, non sans avoir souligné auparavant que les exemples qui suivent se sont renouvelés et répétés dans de nombreuses autres réalisations souterraines que nous ne pouvons pas toutes développer ici.

\section{2}

\section{La recherche sur la réponse en déformation du milieu}

En partant de la considération que la stabilité d'une excavation à court et à long terme est strictement liée à la uformation de l'effet de voûte » et que sa formation et sa position par rapport à la cavité sont signalées par la "réponse en déformation» du milieu à l'action de l'excavation en termes d'importance et de typologie, le besoin se fit résolument sentir de mener des études approfondies sur les rapports entre la modification de l'état de contrainte dans le milieu, provoquée par l'avancement d'un tunnel et la réponse en déformation qui s'ensuit.

Nous estimons qu'il est tout particulièrement indispensable de focaliser l'attention sur la genèse, l'évolution, le contrôle et la stabilisation de la réponse en déformation. A ces fins (Fig. 1):

1) nous avons décidé de consacrer une première période - appelée première phase de la recherche - à l'observation systématique du comportement en déformation du système front de taille-noyau d'avancement et non de la cavité seule, comme l'a proposé et continue de le proposer même aujourd'hui la NATM et les méthodes qui en dérivent;

2) dans une seconde période - appelée deuxième phase de la recherche - sur la base des analyses approfondies des phénomènes d'instabilité observés au cours de l'exécution de nombreux tunnels dans les types de terrains les plus divers et dans les situations les plus disparates de contrainte et de déformation, sur le plan chronologique notamment, nous avons essayé de vérifier l'existence de liens entre le comportement en déformation du système front de taille-noyau d'avancement (extrusion et préconvergence) et celui de la cavité (convergence); 

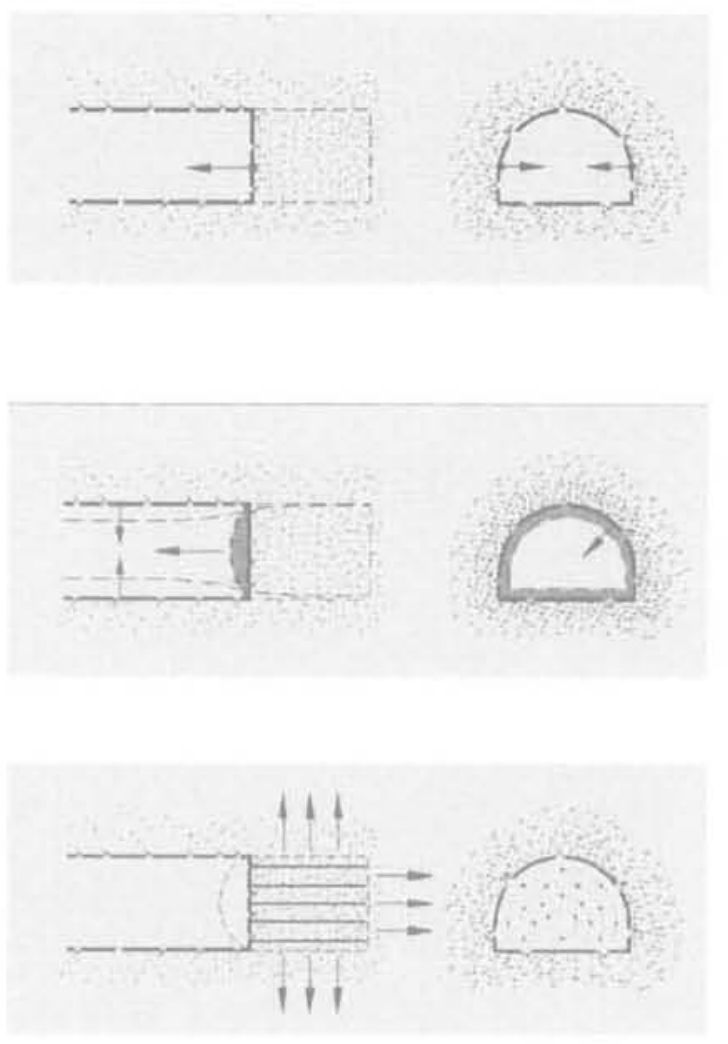

FG. 1 Phases du programme de recherche sur la genèse et l'évolution des phénomènes de déformation du système « front de taille-noyau d'avancement ).

1) Observation systématique du comportement en déformation du système front de taille-noyau d'avancement et non seulement de la cavité:

2) Vérification de l'existence de liaisons entre le comportement en déformation du système front de taille-noyau

d'avancement et de la cavité

3) Vérification du fait qu'en réglant la rigidité du noyau on peut contrôler la réponse en déformation de la cavité. Outline of the research phases.

3) une fois constaté que le comportement en déformation de la cavité est systématiquement conditionné par la rigidité du noyau de terrain au front de taille, dans une troisième période de temps - appelée troisième phase de la recherche - nous avons cherché à vérifier jusqu'à quel point, en agissant sur la rigidité et donc sur la déformabilité du noyau, il était possible de régler et de contrôler la réponse en déformation de la cavité.

\section{1}

\section{Première phase de la recherche}

La première phase de recherche (observation systématique du comportement en déformation du système front de taille-noyau d'avancement) a été menée en faisant des contrôles visuels et par instruments du comportement en déformation des parois d'excavation, en ce qui concerne notamment les phénomènes suivants (Fig. 2):

a) l'extrusion du front de taille. En fonction du matériau et de l'état de contrainte, elle peut se manifester selon des géométries de déformation plus ou moins axisymé- triques (gonflement du front) ou de renversement gravitationnel (rotation du front);

b) la préconvergence de la cavité, c'est-à-dire la convergence du profil théorique en amont du front de taille. Elle dépend strictement des caractéristiques de déformabilité du noyau en relation avec l'état présent des contraintes:

c) la convergence de la cavité qui se manifeste par la réduction de la section théorique d'excavation.

Pour les contrôles expérimentaux, en plus de l'exécution systématique des mesures de convergence de la cavité superficielles et profondes déjà connues, nous avons étudié, mis au point et effectué de nouveaux types de contrôles qui devaient nous permettre d'étudier à fond, dans une section donnée, le comportement en déformation du milieu avant. pendant et après l'arrivée du front de taille et tout particulièrement la zone même du front. En particulier des mesures de préconverqence on été effectuées à partir de la surface avec la mise en cuvre, toutes les fois que la morphologie du terrain et l'importance de la couverture le permettaient, d'instruments multibase pour la mesure des déformations (tassomètres ou sliding micrometres), introduits verticalement dans le terrain à la hauteur de la clef et des reins du tunnel à réaliser.

Dans la plupart des cas, les mesures de préconvergence s'accompagnaient de mesures d'extrusion du noyau d'avancement, réalisées par l'introduction horizontale dans le noyau même d'un sliding micrometer et complétées, pour ces typologies de déformation, par des ratissages topographiques des cibles de référence positionnées sur le front.

Les observations visuelles effectuées systématiquement à l'intérieur de la cavité nous ont permis de leur associer les manifestations d'instabilité localisées soit sur le front soit sur le contour de la cavité.

\section{Deuxième phase de la recherche}

Après avoir défini les typologies de déformation et les manifestations d'instabilité qui peuvent se produire sur le noyau au front de taille et sur le contour de la cavité d'un tunnel, nous nous sommes posé la question de savoir si à travers l'observation des premières il était possible d'être en quelque sorte orientés sur le type et sur l'importance qu'auraient eu les secondes. Nous avons ainsi commencé la deuxième phase de recherche [vérification de l'existence de liajsons éventuelles entre le comportement en déformation du système front de taille-noyau d'avancement $(\rightarrow$ extrusion et préconvergence) et celui de la cavité $(\rightarrow$ convergence)] qui a été menée par l'étude, l'observation et le contrôle dés événements de déformation du front et de la cavité, en tenant tout particulièrement compte de leur importance et de leur succession chronologique en fonction des méthodes, des phases et des cadences d'excavation adoptées au cours des travaux.

Avant de présenter les résultats obtenus dans cette phase expérimentale, il est indispensable d'illustrer les observations faites par quelques exemples significatifs. 

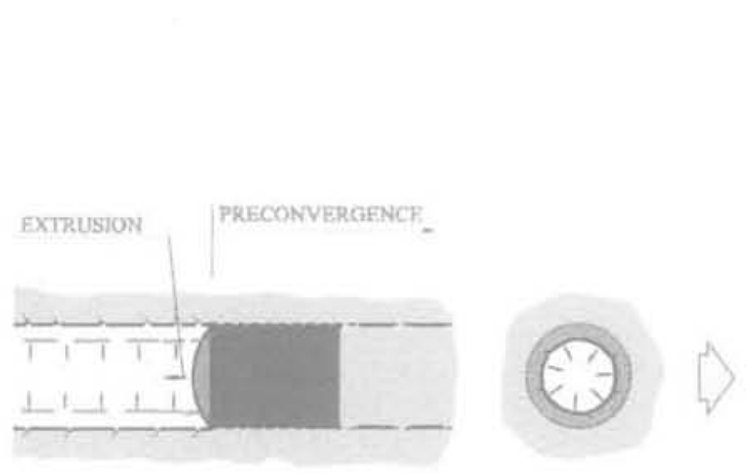

- CONVERGENCE
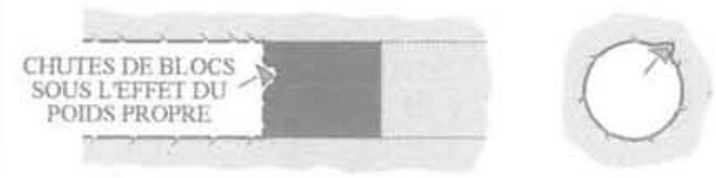

CHUTES DE BLOCS SOUS LEFFET DU POIDS PROPRF
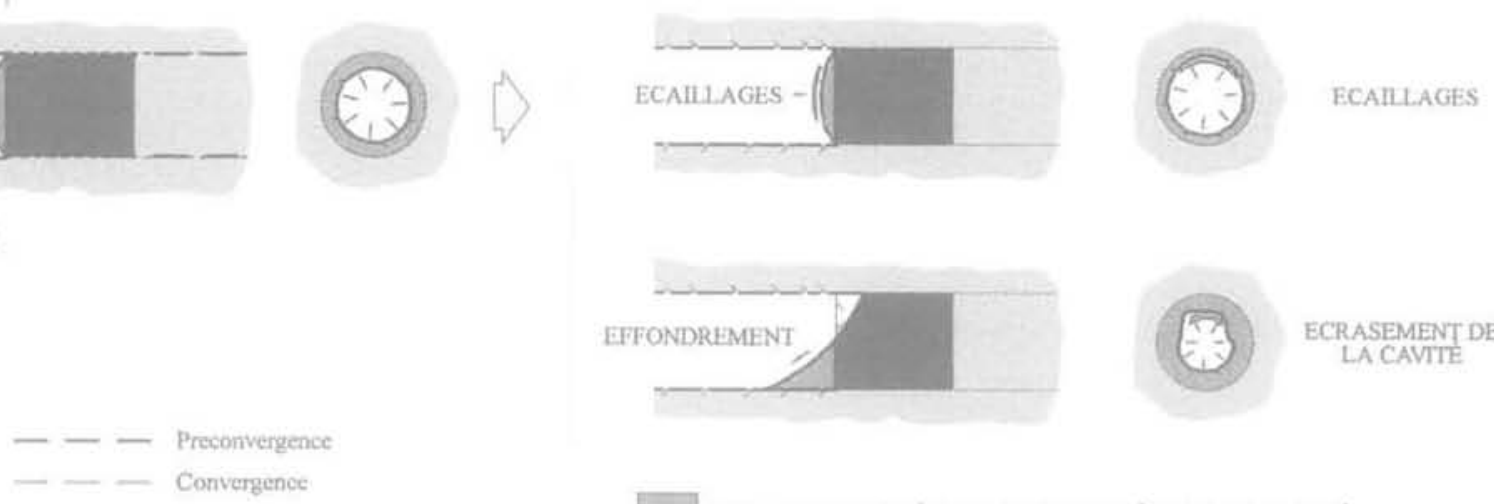

ECRASEMENT DE LA CAVTTE

TERRAIN DINTRUSION À IRAVERS LE PROFLL THEORIQUE DELA CAVIT

HG. 2 Synthèse de la 1 ro phase de recherche : typologies de déformation et manifestations conséquentes de l'instabilité (par instabilité on entend l'intrusion de terrain à travers le profil théorique d'excavation). Outline of the $1^{\text {st }}$ research phase.

\section{Q9.1}

\section{Le tunnel autoroutier du Fréjus (1975)}

Le tunnel de l'autoroute du Fréjus traverse sur 95\% de son tracé $(13 \mathrm{~km}$ de longueur, avec des couvertures atteignant $1700 \mathrm{~m}$ ) une formation métamorphique de calcschistes lithologiquement homogène le long de son tracé.

A l'appui du projet du tunnel, une campagne géologique et géomécanique a été menée dans le tunnel ferroviaire adjacent (réalisée en 1860) et dans les tunnels de service. Les essais de résistance et de déformabilité effectués sur des échantillons de calcschistes indiquaient les paramètres géotechniques moyens suivants :

- angle de frottement interne: $35^{\circ}$;

- cohésion: $3 \mathrm{Mpa}$;

- module élastique : $10000 \mathrm{MPa}$.

Dans le cadre du projet originaire (1975), aucune prévision n'avait été formulée quant au comportement en déformation du tunnel car à l'époque, cela ne relevait pas des pratiques habituelles.

Compte tenu de l'expérience acquise un siècle plus tôt par Sornmeiller au cours de la réalisation du tunnel ferroviaire adjacent, nous avons choisi d'avancer à pleine section en stabilisant immédiatement l'anneau de roche autour de la cavité, sur une épaisseur de $4,5 \mathrm{~m}$ environ, par des boulons actifs à ancrage ponctuel intégrés dans du béton projeté. Le revêtement définitif en béton dont l'épaisseur moyenne était de $70 \mathrm{~cm}$, était coulé par la suite et complétait l'ouvrage.

L'étude des phénomènes de déformation a constitué la partie la plus significative de la campagne d'observations et de mesures pratiquée en plein chantier pour tenir sous contrôle la réponse du massif rocheux face aux interventions de stabilisation opérées. Compte tenu notamment du fait exceptionnel que nous nous trouvions pour la première fois devant un tunnel qui allait être réalisé dans un massif homogène (calcschiste) avec des couvertures variables, soumis à un champ de contraintes croissantes et variant selon la couverture $(0-1700 \mathrm{~m})$.

Jusqu'à 500 m environ de couverture, les contraintes s'exerçaient dans un champ élastique et le tunnel révélait un comportement à front stable, avec des phénomènes de déformation négligeables et des manifestations d'instabilité limitées sur le front de taille et sur la cavité, dues exclusivement à des arrachements de type gravitationnel.

Avec l'augmentation de la couverture et par conséquent l'augmentation de l'état de contrainte, le massif rocheux passait en élastoplasticité, le tunnel adoptait un comportement à front stable à court terme, avec des phénomènes de convergence sur le contour de la cavité de l'ordre de quelques décimètres (convergence diamétrale 10 à $20 \mathrm{~cm}$ ). L'anneau de roche armée collaborait efficacement à la statique du tunnel limitant leur importance et évitant l'apparition des manifestations d'instabilité qui en découlent.

L'avancement, notamment qrâce à la bonne qualité de la roche, continuait sans problèmes, à la vitesse de $200 \mathrm{~m}$ par mois, jusqu'a ce que les travaux soient momentanément arrètés (au P.M. 5172) pour les vacances d'été dans une zone de massif homogène, en présence d'une couverture de $1200 \mathrm{~m}$ environ (Fig. 3).

La station $n^{\circ} 6$ de mesure des convergences, mise immédiatement en place à un mètre du front de taille (P.M. 5 172), présentait, au bout de 15 jours d'arrêt, une déformation maximale de $10 \mathrm{~cm}$ environ. Il s'agissait incontestablement d'une déformation de simple fluage (à charge constante), le front étant resté complètement immobile entre-temps. A la reprise des excavations, la convergence diamétrale, dans la même section, augmentait assez brusquement jusqu'à atteindre des valeurs jamais mesurées auparavant de $60 \mathrm{~cm}$ au bout de trois mois. Puis, poursuivant l'avancement, la 


\begin{tabular}{|c|c|c|c|c|c|c|c|c|c|}
\hline$m \pi$ & " & " & 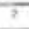 & 8 & $\pi$ & c. & 3 & 3 & 7 \\
\hline netstionst & & & & $*$ & c & & * & & $* \rightarrow$ \\
\hline
\end{tabular}
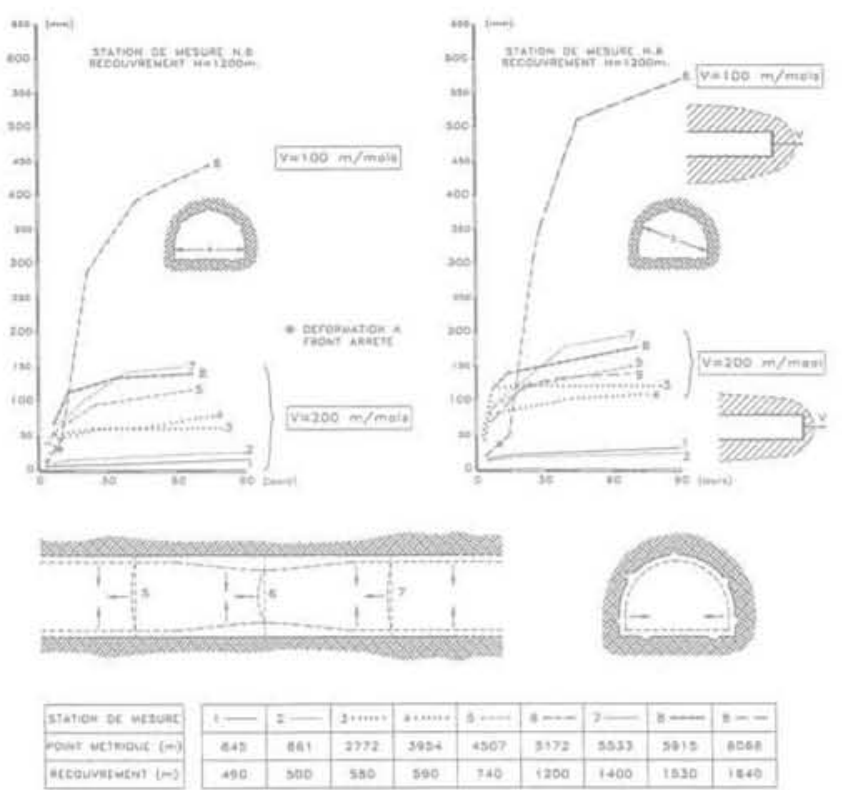

FG. 3

Tunnel autoroutier du Fréjus. Fréjus motorway tunnel.

convergence retrouvait des valeurs normales après quelques dizaines de mètres (convergence diamétrale de $20 \mathrm{~cm}$ environ).

Il convient de préciser qu'avant l'interruption des travaux, la cavité avait été consolidée jusqu'à un mètre du front de taille avec plus de 30 boulons par mètre linéaire mais aucune intervention n'avait eu lieu sur le noyau. Une fois l'avancement repris, l'intervention de stabilisation sur le contour de la cavité s'était poursuivie avec la même intensité et à la mêrne cadence qu'auparavant.

Nous en avons déduit qu'au cours de la fermeture du chantier d'avancement, le noyau de terrain au front de taille, non aidé par des interventions de consolidation, avait eu tout le temps d'extruder en élastoplasticité, amorçant un phénomène de détente par fluage du massif sur son contour (préconvergence) quì a produit à son tour l'augmentation très importante des convergences de la cavité par rapport aux valeurs normales (Fig. 3).

\section{and}

\section{Le tunnel de San Stefano (1984)}

Le tunnel de San Stefano fait partie du nouveau tracé à double voie de la ligne de chemin de fer GênesVintimille, dans le tronçon compris entre San Lorenzo al Mare et Ospedaletti.

L'ouvrage traverse la formation de Flysch à Helmintoïdes caractéristique de la Ligurie occidentale. Il s'agit de schistes argileux et argileux-arénacés avec de minces bancs de grès et de calcaires marneux repliés et intensément fracturés. La composante argilo-schisteuse est fortement laminée. Une zone de transition assez tectonisée marque le passage entre la partie H2 et la partie H1 la plus calcaire-marneuse de la formation (Fig. 4).
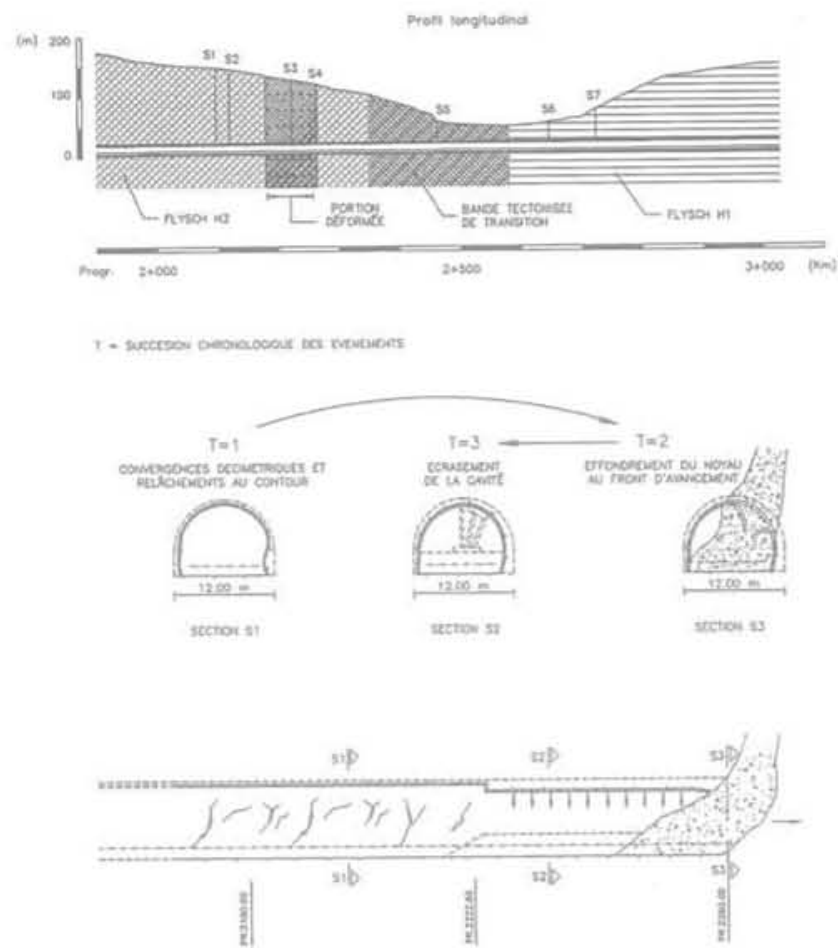

FG.4 Ligne ferroviaire Gênes-Vintimille: portion déformée du tunnel de San Stefano. San Stefano rallway tunnel: out of shape part.

Les essais de résistance effectués en laboratoire sur des échantillons prélevés donnaient des valeurs d'angle de frottement variant entre 20 et 24 degrés avec une cohésion de 1,5 MPa à 0 .

Dans ce cas aussi, lorsque nous avons commencé les travaux, en 1982, aucune prévision n'avait été faite sur le comportement en déformation du tunnel.

Le projet initial prévoyait l'avancement à pleine section, avec la mise en place de cintres et de béton projeté comme revêtement de première phase et d'un anneau de béton de grande épaisseur (jusqu'à $110 \mathrm{~cm}$ ) en tant que revêtement définitif.

Au cours des travaux d'excavation, il a été possible de constater que tant que l'on avançait dans des conditions d'élasticité, les phénomènes en déformation du front et de la cavité étaient tout à fait négligeables. Les manifestations d'instabilité localisées étaient pratiquement absentes (comportement à front stable). Au fur et à mesure que les excavations avançaient et que l'on pénétrait dans une zone intéressée par des états de contrainte résiduels d'origine tectonique, le massif se trouvant dans des conditions d'élastoplasticité, les manifestations en déformation commençaient à entraîner quelques difficultés compte tenu notamment d'importantes poussées dissymétriques dues à la présence dans le terrain de masses rigides dispersées dans la matrice plastique. En même temps, on observait sur le front de taille, des détachements de plaques de matériau, signal sûr et certain de la présence d'un mouvement extrusif, typique d'une situation de front stable à court terme, et avec des convergences dont les valeurs étaient de l'ordre de quelques décimètres.

A un moment donné, la situation de contrainte du massif ayant manifestement évolué jusqu'au champ de rupture, c'était l'effondrement du front de taille tout entier (situation de front instable) suivi, dans l'espace 


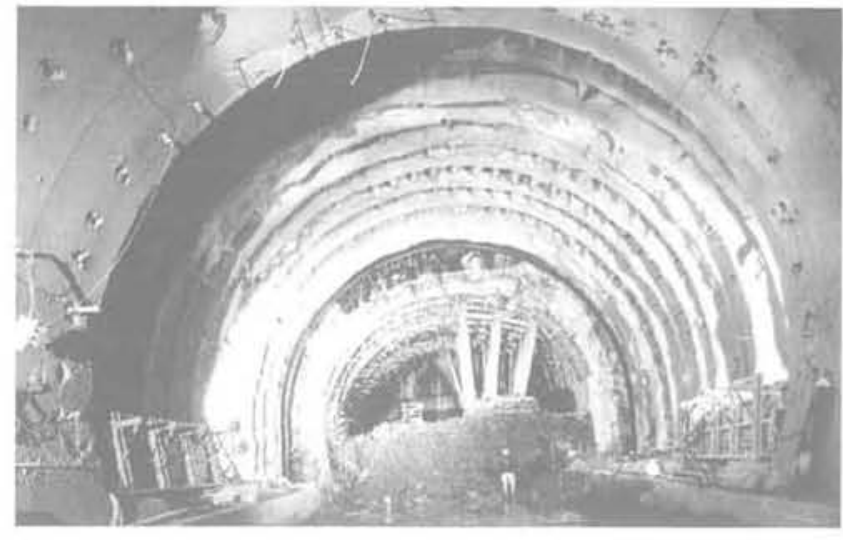

FIG. 5 Tunnel San Stefano:

effondrement de la cavité.

San Stefano tunnel: collapse of the cavity.

de quelques heures, de l'écrasement de la cavité, avec des convergences diamétrales de plus de 2 mètres, même dans la partie déjà stabilisée par des cintres et du béton projeté, sur une longueur très significative (plus de $30 \mathrm{~m}$ en aval du front de taille (Fig. 4 et 5).

Il convient, à ce stade, de souligner que le type de terrain traversé dans les trois situations de contrainte et de déformation citées était fondamentalement le même et que le seul phénomène d'écrasement de la cavité, avec des convergences de l'ordre de 2 mètres, bien que dans une partie de tunnel déjà stabilisé, ne s'est produit en fait que lorsque la contribution de rigidité du noyau au front de taille a fait défaut.

\section{(5)}

\section{Le tunnel du Tasso (1988)}

Le tunnel du Tasso fait partie d'une série de tunnels excavés vers le milieu des années 80 pour la réalisation de la nouvelle ligne «G.V.» Rome-Florence (Fig. 6). La zone où l'ouvrage se situe appartient au bassin lacustre du Valdarno Superiore, constituée de sables limoneux et de limons sabionneux intercalés de strates argilolimoneuses contenant des lentilles et des niveaux sablonneux saturés d'eau.

Initialement, le projet prévoyait d'avancer à demisection, en renforçant les parois de l'excavation par des cintres et du béton projeté. Les cintres étaient fixés au pied par des tirants sub-horizontaux et fondées sur les micropieux ou sur des colonnes de terrain consolidé par jet-grouting.

Initialement, l'excavation - qui se trouvait dans des conditions de front stable à court terme - ne manifesta pas de phénomènes de déformation appréciables, ni du front, ni de la cavité.

Au fur et à mesure que les couvertures et donc l'état de contrainte du milieu augmentaient, du fait notamment des caractéristiques géomécaniques insuffisantes du matériau traversé, on est passé en très peu de temps d'une situation de front stable à court terme à une situation de front instable. Après l'effondrement du front, malgré un avancement en demi-section, il se produisit, pendant une seule nuit, l'écrasement de la cavité avec des convergences diamétrales de l'ordre de 3-4 mètres sur 30-40 mètres de tunnel déjà excavé et protégé par des cintres et du béton projeté (Fig. 6).
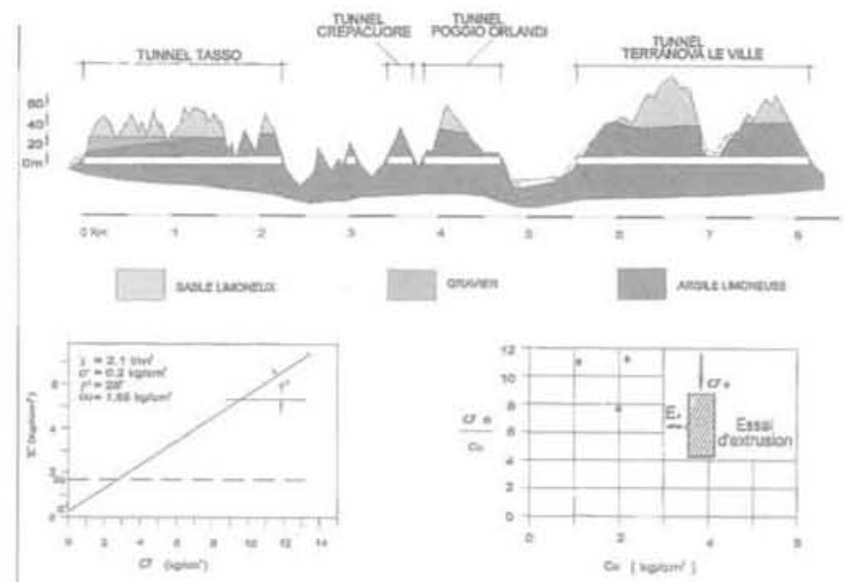

EVENEMENT 1: EFFONDREMENT DU FRONT DE TAILLE
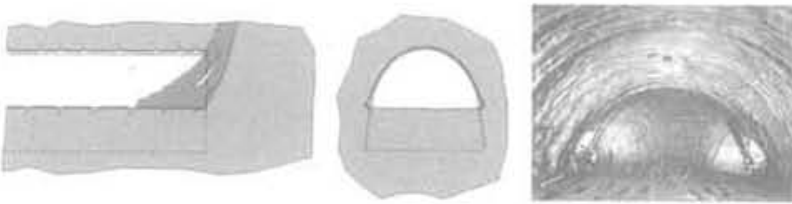

EVENEMENT 2: ECRASEMENT DE U CAVITE
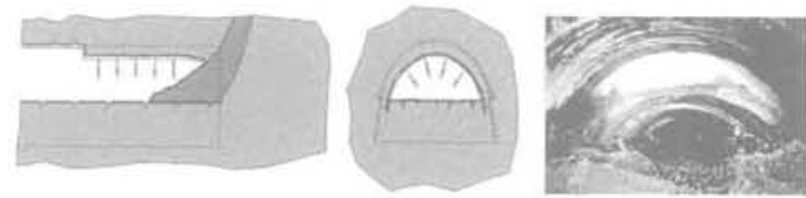

FG. 6 Ligne ferroviaire «G.V.» Rome-Florence. High speed railway line Roma-Firenze.

\section{Résultats de la deuxième phase de la recherche}

L'étude et l'analyse des cas illustrés ainsi que d'autres cas analogues nous ont permis de constater que:

- lorsqu'on avance dans des conditions d'élastoplasticité du massif, il est extrèmement important de ne pas laisser au noyau le temps de se déformer. A cet égard, l'exemple du Fréjus dont nous avons parlé est très significatif puisque dans un massif lithologiquement homogène, l'arrêt de l'avancement du front dans un milieu contraint en champ élastoplastique a comporté, avec la reprise des travaux, une augmentation très importante des phénomènes d'extrusion, de préconvergence et enfin de convergence de la cavité. Une fois l'avancement repris, ces phénomènes reprirent rapidement les valeurs qui avaient été mesurées auparavant. Cela signifie que si nous conservons une cadence d'excavation soutenue et constante, il est possible d'éviter l'amorce des phénomènes d'extrusion et de préconvergence qui constituent l'antichambre des phénomènes de convergence de la cavité qui s'ensuivent;

- l'effondrement du noyau et l'écrasement de la cavité ne se produisent jamais sans que l'un suive l'autre et en particulier sans que le second ne soit précédé du premier. Cette situation, observée plusieurs fois au cours de la réalisation de nombreux tunnels en plus de ceux qui viennent d'être cités, souligne, au-delà de tout doute possible (Fig. 7): 


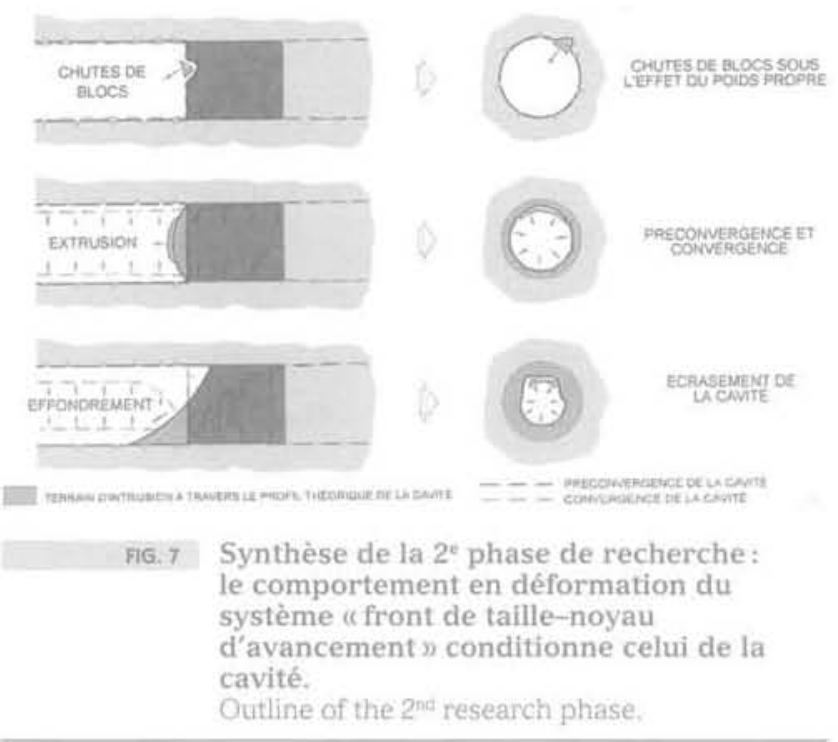

1) l'existence d'un lien étroit entre le phénomène d'extrusion du noyau au front de taille et les phénomènes de préconvergence et de convergence;

2) l'existence de liens étroits entre l'effondrement du noyau d'avancement et l'écrasement de la cavité même déjà stabilisée:

3) que les phénomènes de déformation de la cavité sont toujours chronologiquement postérieurs aux phénomènes qui impliquent le noyau d'avancement dont ils dépendent.

Ce que nous avons observé dans la seconde phase de recherche montre clairement le besoin de faire en sorte que l'effet de voûte - dont la formation, nous le savons, conditionne la stabilité du tunnel -s'amorce déjà en amont du front de taille pour continuer à être opérationnel dans une section donnée, même après que le front se sera éloigné d'elle.

\section{3}

\section{Troisième phase de la recherche}

Les résultats de la seconde phase de la recherche renforçaient notre impression que la rigidité du noyau d'avancement d'un tunnel jouait un rôle déterminant dans sa stabilité à court et à long terme. Puisque le comportement de la cavité répondait corrolairement à la rigidité du noyau, il est apparu logique d'envisager la possibilité d'utiliser le noyau comme instrument de stabilisation pour le tunnel en cours d'avancement, après confirmation de la possibilité de modifier sa rigidité par des interventions appropriées.

Nous avons donc travaillé sur la rigidité du noyau pour vérifier jusquà quel point ceci pouvait permettre de contrôler la réponse en déformation de la cavité. Pour ce faire, il nous a fallu étudier et mettre au point des technologies nouvelles pour agir sur le noyau, pour faire varier sa résistance au cisaillement ainsi que les caractéristiques de déformabilité: c'est l'origine des systèmes de conservation directs, indirects et mixtes.

Ces nouvelles idées ont été expérimentées au cours de la réalisation de plusieurs tunnels dans des condi- tions de contrainte et de déformation difficiles. Voyons tout particulièrement ce qui a été fait dans un chantier expérimental particulièrement significatif.

\section{7) \\ Le tunnel de San Vitale (1991)}

Le tunnel de San Vitale a 4200 mètres de longueur, avec des couvertures variables jusqu'à $150 \mathrm{~m}$. Il fait partie du projet de doublement et de renforcement de la ligne de chemins de fer Caserta-Foggia entre les gares d'Apice et de Vitulano, aux portes de Benevento.

Le tracé se développe dans des terrains appartenant à deux unités litho-stratigraphiques. L'œ Unità di Altavilla s et l'œUnità delle Argille Varicolorin) (voir profil de la Fig. 8),

L'Unità di Altavilla, moyennement tectonisée, est constituée d'alternances de niveaux de sables, parfois même peu cimentés, et de niveaux d'argiles marneuses et d'argiles silteuses.

L'Unità delle Argille Varicolori qui se trouve audessous est constituée de deux différents lithotypes. L'un essentiellement argileux-marneux, l'autre essentiellement calacaréo-marneux. Tous deux ont fait l'objet d'une tectonisation intense qui leur a conféré une structure écailleuse désordonnée et chaotique et les a rendus extrêmement altérables, donnant lieu à une fragmentation très menue des parties lithoïdes les plus compétentes. Actuellement, celles-ci ont pris la forme de massifs isolés enfermés dans une matrice argileuse ou argilo-marneuse et non en niveaux continus comme c'était le cas initialement. Le terrain se présente réduit en menus fragments qui tendent à lui conférer le comportement d'un massif incohérent, avec des boucles de matériaux pierreux pliés par les forces tectoniques et dispersés dans la matrice argileuse dont les écailles ne sont plus de l'ordre du décimètre mais du millimètre, ce qui la rend très sensible à l'humidité de l'air.

\section{magaz \\ Très rapidement, l'histoire de l'excavation}

L'excavation du tunnel a commencé en mars 1986. Le projet initial reposait sur des critères de la NATM. I prévoyait pour tout le tunnel, l'excavation en classe $\mathrm{Vb}$ en sections divisées, avec l'exécution d'un boulonnage radial en aval du front de taille. La fermeture de l'anneau résistant de la première phase était réalisée à $30 \mathrm{~m}$ environ de distance du front de taille. Grâce à ce système, il a été procédé par fronts opposés jusqu'à la fin de 1988, dans un segment qui se caractérisait par la présence du faciès marno-arénacé de l'Unità di Altavilla, avec des couvertures de l'ordre de 30 à 40 mètres. Comme on le voit sur la figure 9 , la méthode d'avancement mise en place ne prévoyait aucun type de confinement du terrain en amont du front de taille. Par conséquent, aussitôt arrivés dans les arqiles écailleuses. il se produisait des phénomènes d'extrusion très visibles du noyau d'avancement doublés de phénomènes importants de préconvergence qui se répercutaient immédiatement en aval sur la demi-section avec des convergences radiales importantes, jusqu'à des valeurs de l'ordre de $1 \mathrm{~m}$, qui compromettaient complètement l'épaisseur utile pour le revêtement en béton, obligeant l'arrêt les travaux d'excavation. 

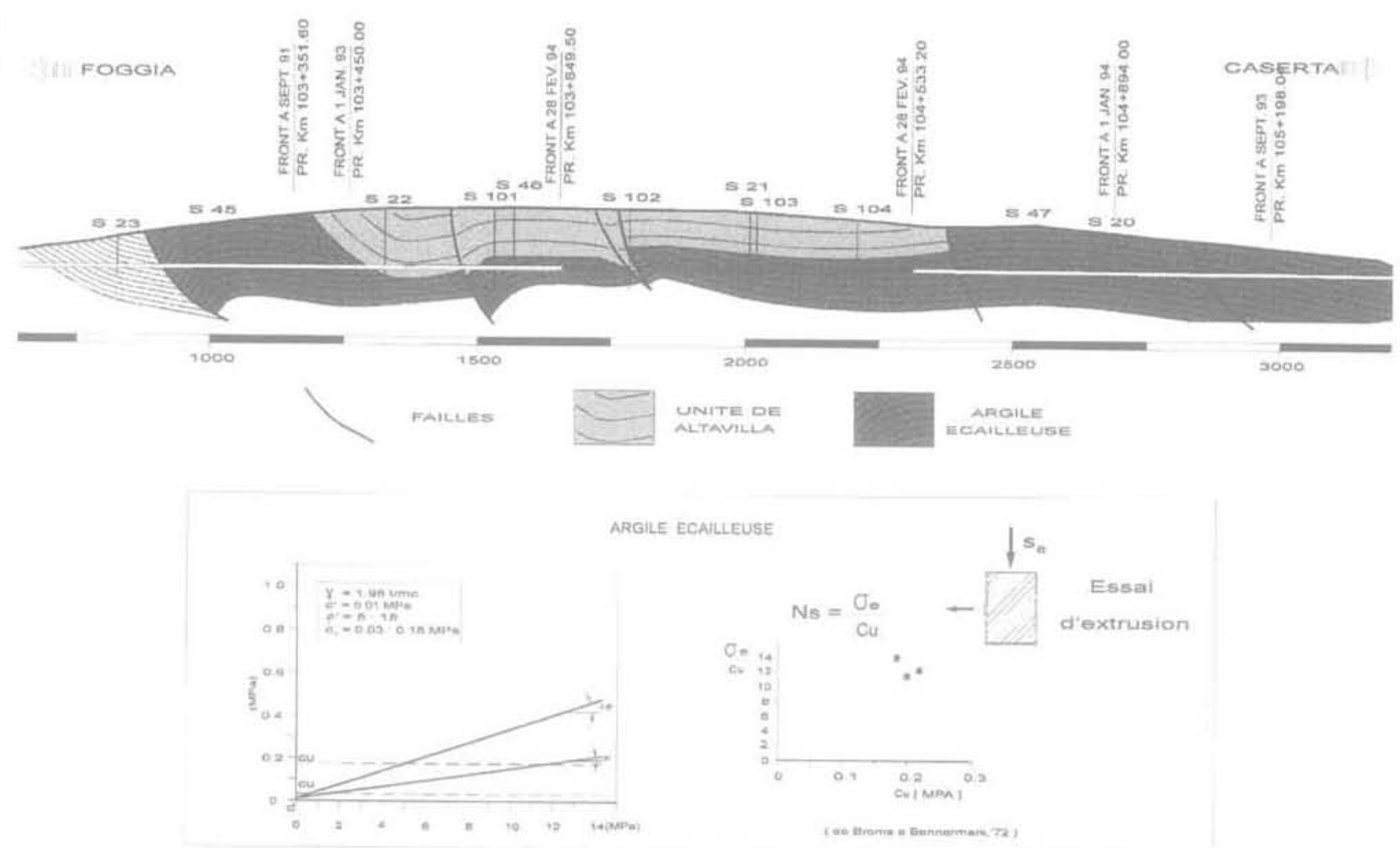

FG. 8 Ligne ferroviaire Caserta-Foggia : phase de reconnaissance au tunnel de San Vitale. Caserta-Foggia railway line: survey phase at San Vitale tunnel.

A la suite de cela, en 1989, un nouveau projet fut élaboré, spécialement pour le segment dans le argiles écailleuses. L'avancement était prévu encore par demisection, avec emploi de tubes métalliques perforés, de tirants radiaux prétendus au niveau des reins et de tubes en résine armée de fibres de verre sur le front de taille. L'exécution d'un radier provisoire complétait l'intervention de la première phase (Fig. 9).
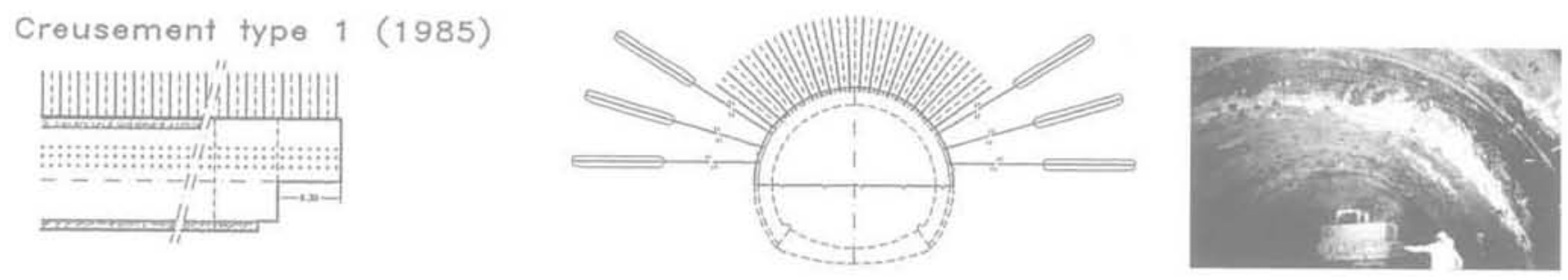

Creusement type 2 (1990)
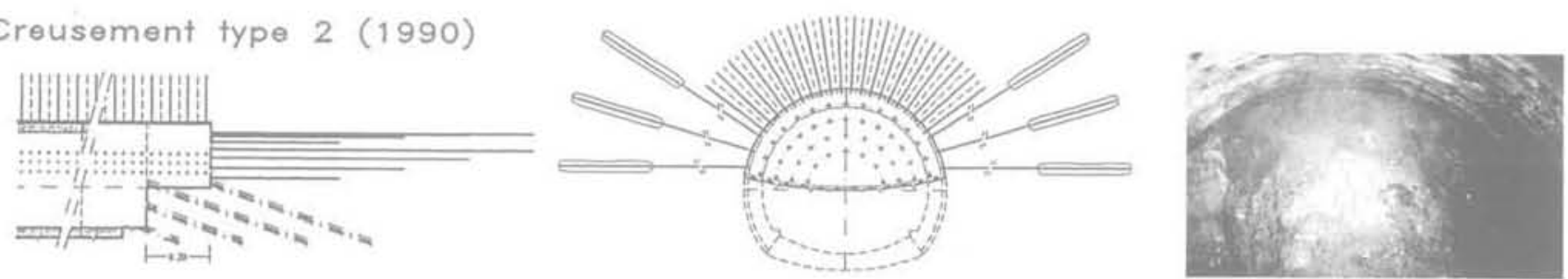

Creusement type 3 (1990)
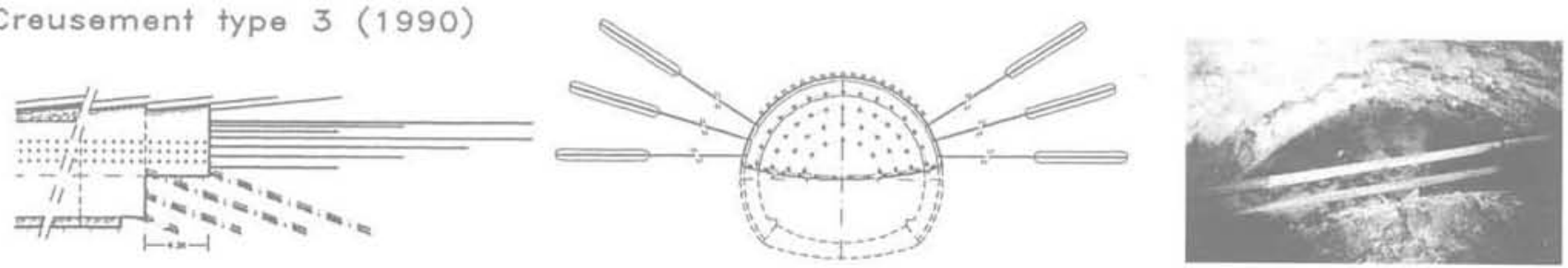

FIG.9 Ligne ferroviaire Caserta-Foggia - Tunnel de San Vitale (phase de thérapie). Caserta-Foggia railway line - San Vitale tunnel (therapy phase). 
L'introduction du renforcement du noyau, effectuée par ailleurs selon des procédures et des critères non corrects, n'avait aucun effet efficace. Les résultats étaient désastreux tant du point de vue de la production ( $80 \mathrm{~m}$ seulement d'avancement en un an) que du point de vue statique. Le court segment excavé était effectivement assujetti à des chutes de matériau constantes, à des convergences élevées, à des ruptures du revêtement de la première phase, etc.

Au cours de toute l'année 1991, aucun avancement ne fut fait. Le Groupe d'entreprises COFERI, en accord avec le client (les Chemins de fer italiens) décidait de confier le projet à Rocksoil S.p.A. de Milan qui, compte tenu des indications fournies par le programme de recherche appliqué jusqu'alors, abandonnait totalement tous les projets précédents, adoptant pour les $1800 \mathrm{~m}$ de tunnel restants un nouveau critère d'avancement dont les principes reposent sur le contrôle des phénomènes de déformation au moyen du raidissement du noyau au front de taille, produisant donc des actions de préconfinement de la cavité.

\section{2,23}

\section{Phase de reconnaissance pour le tunnel de San Vitale}

Avant de commencer le nouveau projet, nous avons pensé qu'il était utile de procéder à une caractérisation géotechnique plus approfondie des matériaux impliqués par les excavations. En plus des essais habituels de laboratoire qui nous ont permis de construire les courbes intrinsèques du matériau et qui ont révélé la grande capacité qu'avaient ces terrains d'absorber rapidement l'humidité et donc de réduire considérablement la résistance au cisaillement (Fig. 10), nous

ESSAIS DE RESISTANCE

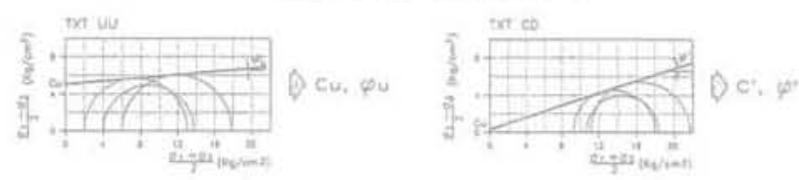

ESSAIS DE DEFORMABILITE
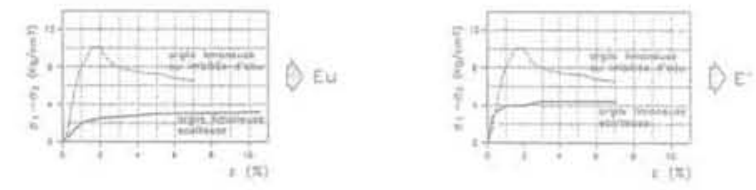

ESSAIS DE SENSIBILITE A L'HUMIDITE
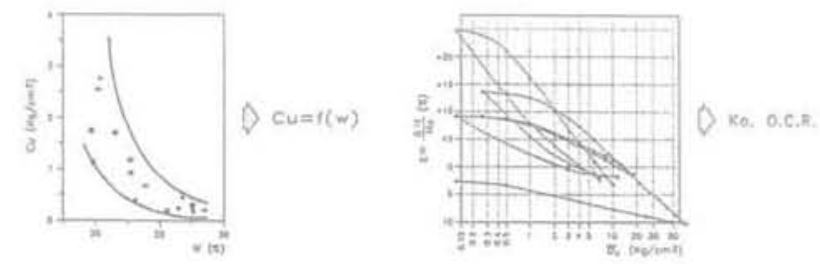

FG. 10 Ligne ferroviaire Caserta-Foggia - Tunnel de San Vitale: essais géotechniques (phase de reconnaissance). Caserta-Foggia railway line-San Vitale tunnel geotechnical tests (survey phase). avons procédé à différents essais d'extrusion à vitesse de charge constante, en cellule triaxiale et aussi en centrifugeuse.

Les deux derniers, conçus et mis au point par Rocksoil S.p.A. de Milan, pour être utilisées spécialement au cours de la phase de diagnostic, permettent d'étudier la future réponse en déformation du système front de taille-noyau d'avancement, simulant en laboratoire sur des bases vraisemblables l'avancement de l'excavation d'un tunnel dans quelque état de contrainte que ce soit.

Tout particulièrement dans les essais en cellule triaxiale, l'échantillon de terrain est introduit dans la cellule en reconstituant l'état de contrainte initial du massif. Grâce à la pression d'un fluide, cet état de contrainte est exercé également à l'intérieur d'un volume cylindrique particulier (qui simule le tunnel dans la zone du front de taille) préparé avant l'essai à l'intérieur de l'échantillon et coaxial par rapport à lui.

En conservant l'état de contrainte sur le contour de l'échantilion et en réduisant progressivement la pression du fluide à l'intérieur du volume cylindrique, nous simulons la modification de contrainte au front provoquée par l'excavation et nous obtenons une évaluation de l'importance du phénomène d'extrusion au front de taille en fonction du temps.

Il est particulièrement utile de se servir des essais d'extrusion en centrifugeuse lorsqu'il est nécessaire de prendre en considération l'effet de la gravité. Ces essais permettent, d'une part, de considérer l'effet des pressions géostatiques agissant sur le contour de l'excavation même dans le cas de couvertures moyennes ou hautes et, d'autre part, ils nous permettent de suivre méticuleusement, grâce à des transducteurs de pression et de déplacement correctement introduits à l'intérieur du modèle, le comportement du phénomène d'extrusion dans le temps.

Le lecteur intéressé peut trouver une illustration plus ample des essais d'extrusion en centrifugeuse effectués pour la réalisation du tunnel de San Vitale dans le mémoire [10] cité dans la bibliographie.

Les essais d'extrusion, intégrés dans des modèles mathématiques simples aux éléments finis, sont extrêmement utiles même pour l'étalonnage des paramètres géomécaniques $(c, \varnothing$, E) à utiliser dans les phases sujvantes de diagnostic et de thérapie.

Pour compléter cette phase de reconnaissance nous avons effectué in situ des essais d'extraction des tubes en résine armée des fibres de verre du front pour déterminer la résistance limite d'adhérence entre le mortier de cimentation et le terrain, à la profondeur du projet.

Les résultats de ces essais, onéreux et difficiles à exécuter, ont été confrontés et intégrés à ceux précédemment obtenus dans des terrains analogues.

\section{4}

\section{Phase de diagnostic pour le tunnel de San Vitale}

Dans la phase de diagnostic, l'étude des résultats des essais d'extrusion et des lignes caractéristiques du tunnel penchait, en l'absence d'interventions de stabilisation et de contrôle des phénomènes de déformation, en faveur d'un comportement à front résolument instable (Fig. 11 et 12). La situation apparaissait encore plus compromise car, comme cela a été montré par les 
a- Exagi dinxtrusion à vitesse de chargenaent contebiad

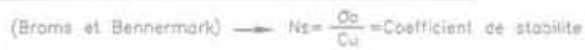
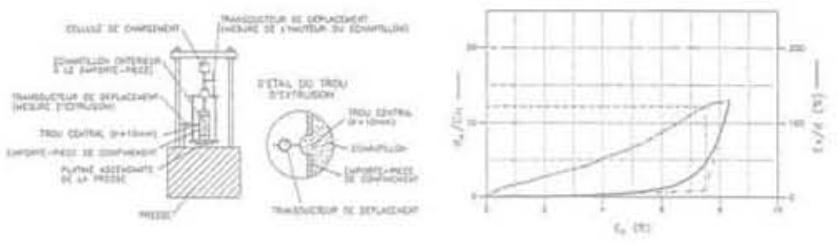

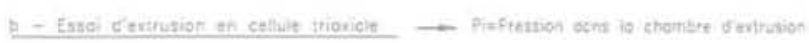
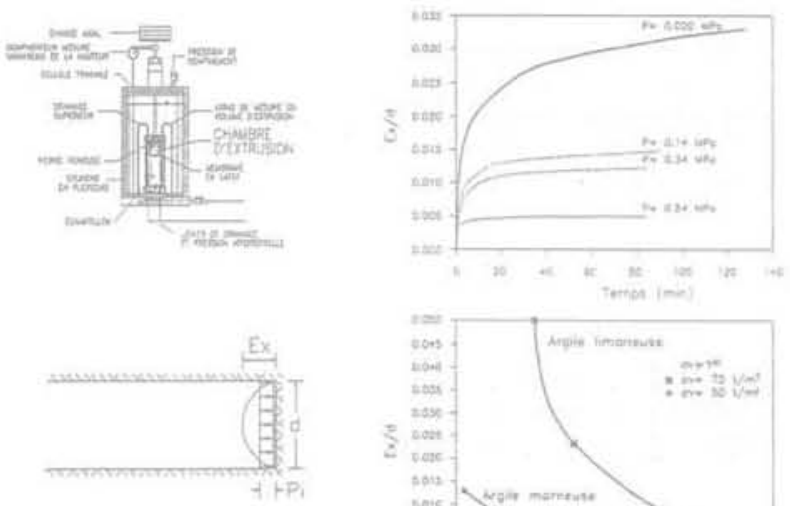

$c_{5}=\gamma=$

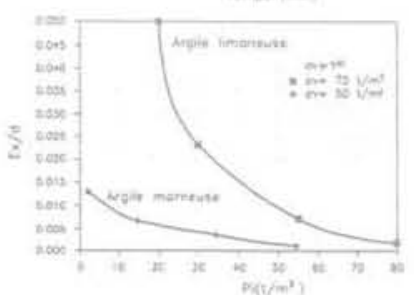

FG. It Ligne ferroviaire Caserta-Foggia - Tunnel de San Vitale : essais d'extrusion (phase de diagnostic).

Caserta-Foggia railway line - San Vitale tunnel: extrusion test (diagnostic phase).

essais d'extrusion en laboratoire, à la suite du relâchement du terrain du fait de l'excavation, il se produisait sur le contour de la cavité une variation rapide de la teneur en eau de l'argile suivie d'une réduction du facteur de stabilité à l'extrusion.
Les essais d'extrusion étaient également modélisés numériquement avec des programmes aux éléments finis pour étalonner d'une part les paramètres de résistance et de déformabilité en fonction des états de contrainte initiaux et de l'autre, pour évaluer qualitativement le type probable de cinématique attendu au front de taille, le volume de terrain impliqué par le phénomène d'extrusion et l'importance des charges mobilisées.

\section{rasat}

\section{Phase de thérapie pour le tunnel de San Vitale}

Dans la phase de thérapie, compte tenu des résultats de la phase de diagnostic, des caractéristiques des tèrrains à creuser et des expériences acquises dans des cas analogues, nous avons choisi d'intervenir en amont du front de taille en empêchant d'une part la diminution de la contrainte principale mineure sur le noyau et de l'autre, en essayant d'amorcer artificiellement l'effet de voûte que le terrain n'aurait pas réussi à mobiliser à lui tout seul.

Nous avons donc projeté une intervention de conservation de type mixte qui répondrait à cette double fonction (Fig. 13) et qui comprendrait:

- un renforcement du noyau d'avancement par des tubes en résine armée de fibres de verre à adhérence améliorée;

- un prérenforcement du contour de la cavité par un écran de matériau cimenté réalisé selon la technique du prédécoupage mécanique;

- des drainages:

- un radier en béton armé coulé derrière le front de taille.

L'intervention de la première phase était complétée par un revêtement de cintres et de béton projeté renforcé de fibres d'acier alors que le revêtement en béton de la seconde phase était coulé aussitôt après.

Dans le cadre du projet, nous avons prévu également une solution alternative à celle du prédécoupage,
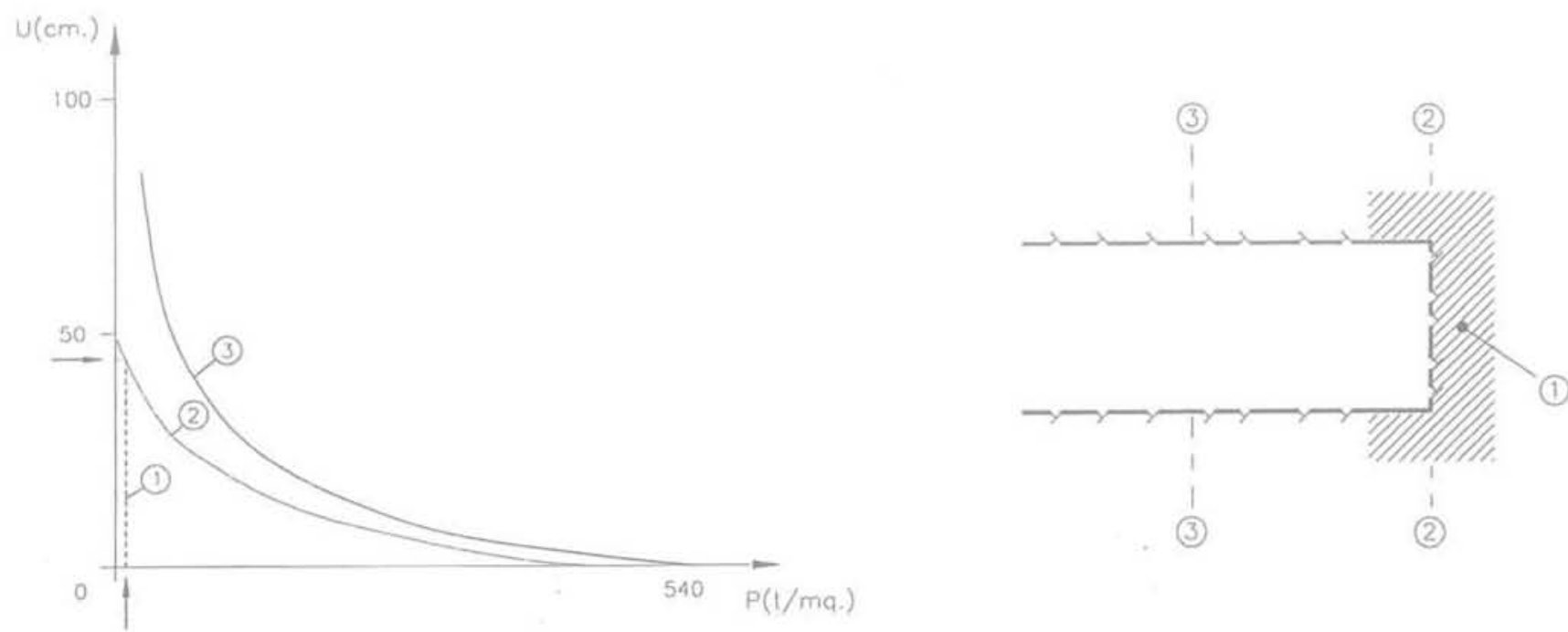

FIG. 12 Ligne ferroviaire Caserta-Foggia - Tunnel de San Vitale : étude par les lignes caractéristiques (phase de diagnostic).

Caserta-Foggia - San Vitale tunnel: caracteristic lines study (diagnostic phase). 

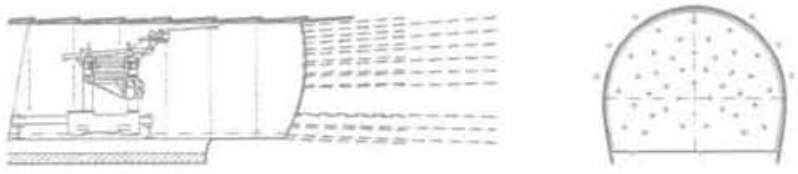

SOLUTION BASE

(PRÉDÉCOUPAGE MÉCANIQUE + VTA)
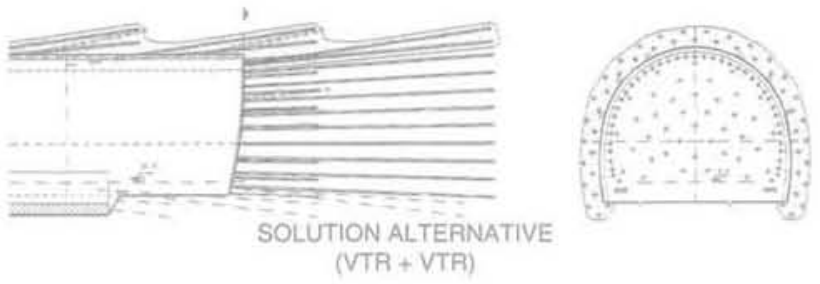

(VTR + VTR)

F16.13 Ligne ferroviaire Caserta-Foggia - Tunnel de San Vitale: phase de thérapie.

Caserta-Foggia - San Vitale tunnel: therapy phase.

prévoyant le remplacement des tuiles de béton projeté renforcé de fibres par une anneau de terrain renforcé par des tubes en résine armée de fibres de verre, équipés pour l'exécution d'injections de recompression et injectés à haute pression (Fig. 14).

Le dimensionnement des interventions de clouage au front, en termes d'intensité, de profondeur et de géométrie, a été effectué, dans une première approximation, par deux méthodes différentes. Une méthode

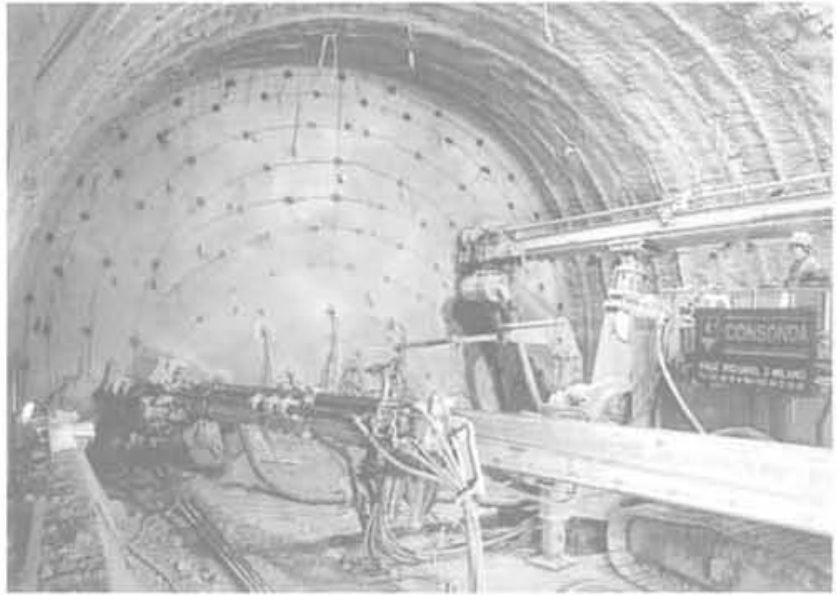

FG.14 Tunnel de San Vitale: avancement par la solution alternative.

San Vitale tunnel: work with the alternate solution.

expérimentale, basée sur l'interprétation des essais d'extrusion et une méthode théorique, basée sur la théorie des lignes caractéristiques (Fig. 15).

Dans le premier cas, sur les diagrammes obtenus par les essais d'extrusion, nous avons défini la pression de préconfinement nécessaire à la stabilisation du front. L'intensité de l'intervention (nombre de tubes en fibre de verre à mettre en ceuvre pour garantir cette pression) a été donc évaluée sur la base des résultats
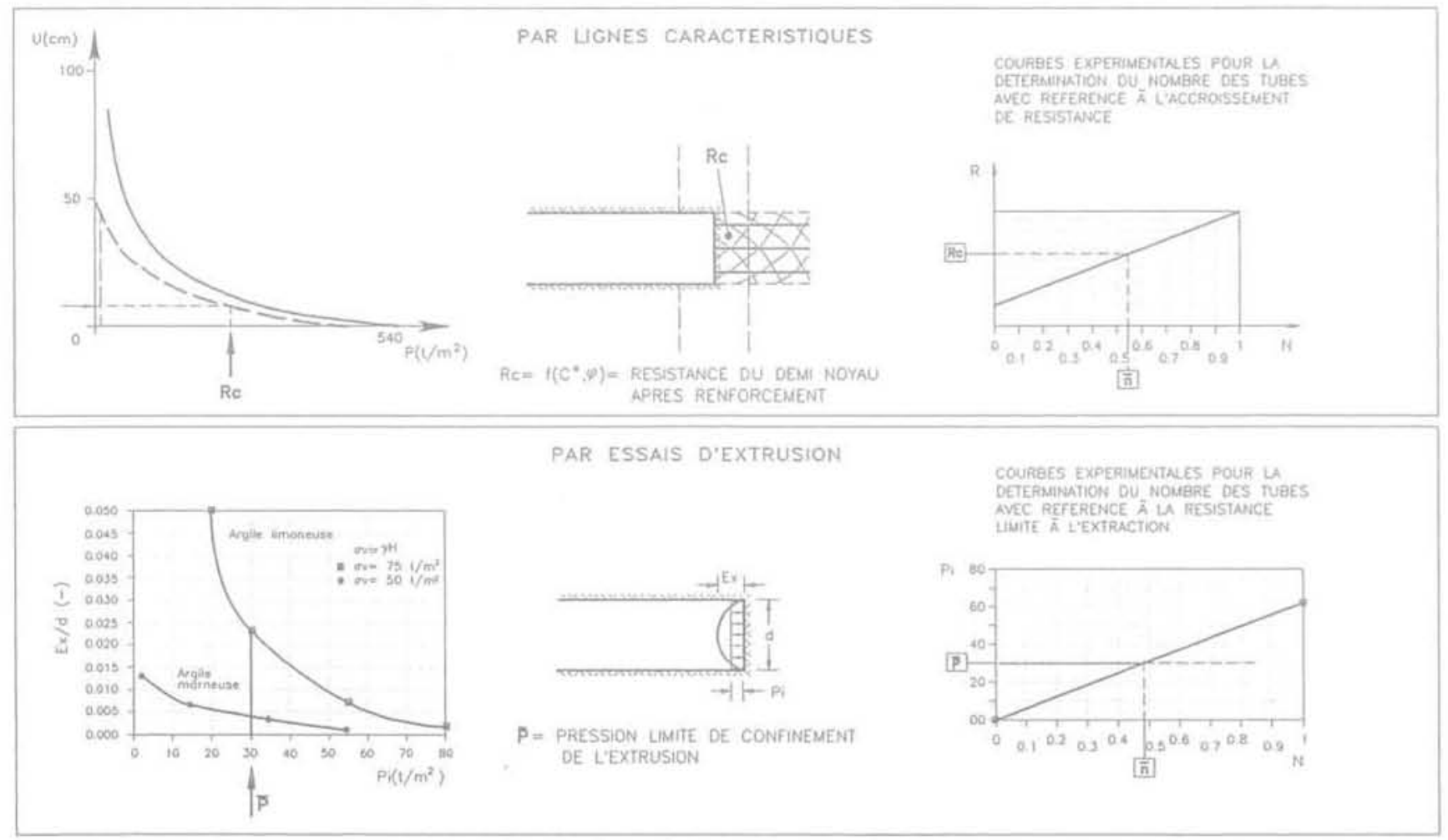

FG. 15 Évaluation de l'étendue du renforcement du noyau pour éviter son instabilité (phase de thérapie). Size assessment of the nucleus reinforcement to avoid instability (therapy phase). 
des essais d'extraction. Lorsqu'ils n'étaient pas disponibles, des hypothèses fiables ont été avancées sur la base d'essais faits dans des terrains analogues.

Des évaluations analogues ont été faites avec la méthode des lignes caractéristiques en tenant compte, d'une manière simplifiée, de l'effet du clouage du front de taille dans le calcul de la ligne caractéristique du noyau. Les deux approches ont fourni des résultats comparables entre eux et confirmé l'analogie conceptuelle existante.

La profondeur de l'intervention (longueur des tubes) a été enfin définie en fonction des mécanismes cinématiques révélés par la simulation numérique des essais d'extrusion.

Pour éliminer les simplifications que nous sommes obligés d'adopter lorsque nous évaluons l'effet de préconfinement exercé par les tubes en résine armée de fibres de verre au front de taille, nous mettons au point un type d'essai d'extrusion en centrifugeuse qui fournira une évaluation directe de l'intensité de l'intervention de clouage nécessaire.

Enfin, la vérification des solutions adoptées et la mise au point de la géométrie de l'intervention ont été effectuées à l'aide d'un modèle numérique tridimensionnel aux éléments finis en domaine non linéaire.

Le modèle a largement confirmé l'importance du contrôle exercé par le noyau d'avancement sur la statique de la cavité. Il a souligné que les cinématiques activées dans la phase d'excavation peuvent être contrôlées en agissant sur l'intensité des interventions de renforcement du noyau.

En définitive, l'étude de thérapie soulignait, pour la solution de base, le besoin de renforcer le noyau d'avancement au moyen de 50 tubes en résine armée de fibres de verre $\varnothing 40 / 10$ de $18 \mathrm{~m}$ de longueur. Pour la solution alternative, l'étude confirmait la possibilité de remplacer les tuiles du prédécoupage par une couronne de terrain renforcé par 49 tubes en résine armée de fibres de verre $\varnothing 60 / 40$, équipés de vannes pour l'exécution d'injections de recompression.

\section{6}

\section{Phase opérationnelle pour le tunnel de San Vitale}

Rassurés par le résultat des vérifications effectuées grâce au calcul numérique, nous avons procédé à l'application pratique des interventions choisies dans la phase conceptuelle, dans le cadre des sections type prévues (Fig. 13).

Les 300 premiers mètres de tunnel ont été excavés avec de bons résultats, en adoptant la solution de base. Par la suite, l'interception le long du tracé d'intercalations calcaires importantes souvent doublées d'amples replis d'origine tectonique, rendait difficile le fraisage de l'arc de prédécoupage, créant des problèmes inacceptables de géométrie et de statique de la voûte. Ne pouvant plus compter sur la collaboration de l'anneau de protection sur le contour de la cavité, constitué par le prédécoupage mécanique car sa continuité aurait fait défaut, nous avons décidé de passer à la solution alternative qui donnait des garanties de continuité par l'emploi du prérenforcement à l'aide de tubes en résine armée de fibres de verre, même sur le contour de la cavité.

L'adoption de la nouvelle section type nous a permis d'augmenter, par des mesures opérationnelles appropriées, les productions déjà bonnes que nous obtenions avec le prédécoupage mécanique (Fig. 16).

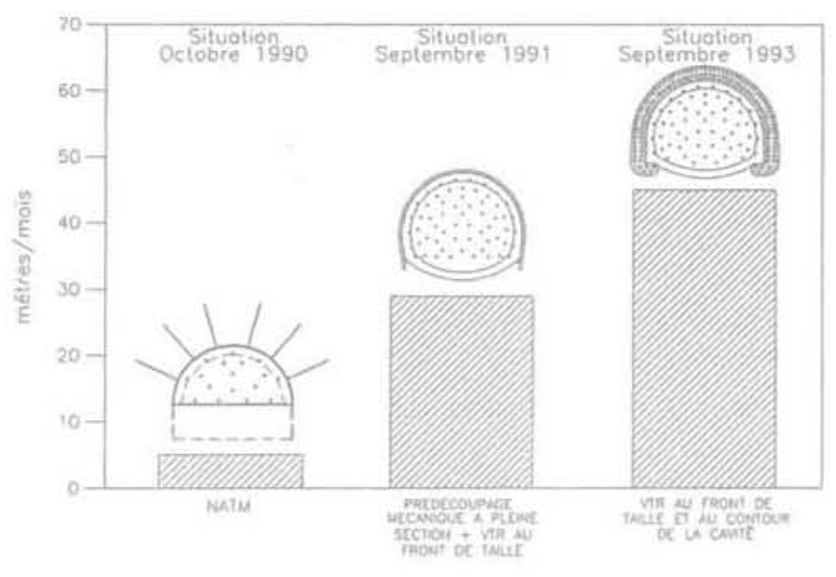

FG. 16 Ligne ferroviaire Caserta-Foggia - Tunnel de San Vitale: productions par typologie d'avancement.

Caserta-Foggia railway line - San Vitale tunnel: production with different methods.

Nous avons constaté que dans des situations particulièrement critiques, le renforcement du noyau seul peut ne pas suffire à s'opposer efficacement aux phénomènes de déformation, même en intensifiant le traitement.

Nous avons bien vu que ces situations peuvent être surmontées:

- en travaillant sur la forme du noyau afin de créer dans l'avancement une bande de terrain renforcé sur le contour du noyau;

- en opérant éventuellement par des interventions radiales de renforcement sur le contour de la cavité, dimensionnées pour absorber les convergences résiduelles que le noyau n'est pas capable d'empêcher à lui tout seul.

A theure actuelle, l'excavation du tunnel de San Vitale se fait avec la section type alternative, sans rencontrer de difficultés.

\subsubsection{7.}

\section{Phase de vérification en cours de chantier du tunnel de San Vitale}

Le système d'avancement adopté dont l'objectif est de créer l'effet de voûte en anticipation sur l'excavation, exige, pour vérifier son comportement, le contrôle continu des phénomènes de déformation. Cela permet au système lui-même d'être mis au point en temps utile, en fonction de son évaluation face aux interventions de stabilisation opérées. Le contrôle prévoit l'exécution de:

- mesures tassométriques de la surface, dans les segments à couverture réduite, opérées de manière à pouvoir connaître le comportement des préconvergences et des convergences en amont et en aval du front de taille du tunnel à la hauteur de la station de mesure; - mesures d'extrusion du noyau au front de taille; - mesures de convergence de la cavité par bande d'acier Invar;

- mesures de pression au contact entre les structures de revêtement et le massif environnant;

- mesures au moyen de vérins plats à l'intrados du revêtement définitif en béton pour les relevés de l'état de contrainte. 
Naturellement, à ces contrôles de base sont associés d'autres contrôles lorsque les particularités de la situation l'exigent.

Dans le tunnel de San Vitale, nous avons notamment fait, pour la toute première fois dans I'histoire de la construction des tunnels, des mesures systématiques et simultanées d'extrusion et de convergence.

Les mesures d'extrusion du front de taille, effectuées par ISMES S.p.A. de Bergame au moven de l'introduction d'un sliding micrometer dans le noyau d'avancement, ont montré des valeurs moyennes de près de $2 \mathrm{~cm}$ contre les $20 \mathrm{~cm}$ mesurés en 1990 (Fig. 17). Les mesures de convergence à leur tour ont montré des valeurs moyennes de $7 \mathrm{~cm}$ seulement contre les $100 \mathrm{~cm}$ et davantage observés en 1990. La réduction significative des valeurs mesurées démontre l'efficacité de la nouvelle approche conceptuelle.

Ce qui est particulièrement intéressant, c'est le comportement des extrusions et des convergences à l'intérieur d'un cycle complet de travaux comportant le renforcement du noyau avec des tubes en résine sur une profondeur de $18 \mathrm{~m}$ et l'excavation d'avancement sur une profondeur minimale de 6 , et maximale de $9 \mathrm{~m}$. Notons à cet égard que les profondeurs d'avancement sont définies au fur et à mesure, en fonction du comportement et de l'importance des convergences de la cavité qui ne doivent pas dépasser le seuil établi par le concepteur afin de maintenir la réponse en déformation le plus possible dans le domaine élastique. La lecture des diagrammes extrusion-convergence de la figure 18 (qui se réfèrent à un segment de tunnel excavé dans un terrain particuliè- rement médiocre) montre que l'avancement du front, au fur et à mesure de la réduction à $10,5 \mathrm{~m}$ de la profondeur du noyau initialement renforcé à $18 \mathrm{~m}$ et par conséquent de la réduction de sa rigidité moyenne, est suivi d'un comportement en déformation du noyau même (extrusion) et de la cavité en aval du front dé taille (convergence) qui passe progressivement du domaine élastique vers l'élastoplastique. Les courbes de convergence passent notamment d'un comportement initial typique d'une situation qui évolue rapidement vers la stabilité à des comportements qui révèlent une difficulté croissante des phénomènes de déformation à se stabiliser.

La convergence de la cavité apparaît ainsi comme un signal très important pour le projeteur pour choisir le moment où il sera nécessaire d'arrêter l'avancement pour effectuer un nouveau renforcement et rétablir la profondeur minimale du noyau renforcé pour maintenir le massif dans le domaine élastique.

\section{4}

\section{Résultats de la troisième phase de la recherche}

L'étude et les expériences faites dans le tunnel de San Vitale ont montré, d'une part, l'existence d'un lien étroit entre les phénomènes de déformation qui se produisent à l'intérieur du noyau d'avancement du tunnel (extrusions) et, de l'autre, ceux qui se produisent plus tard sur le contour de la cavité, en aval du front de taille
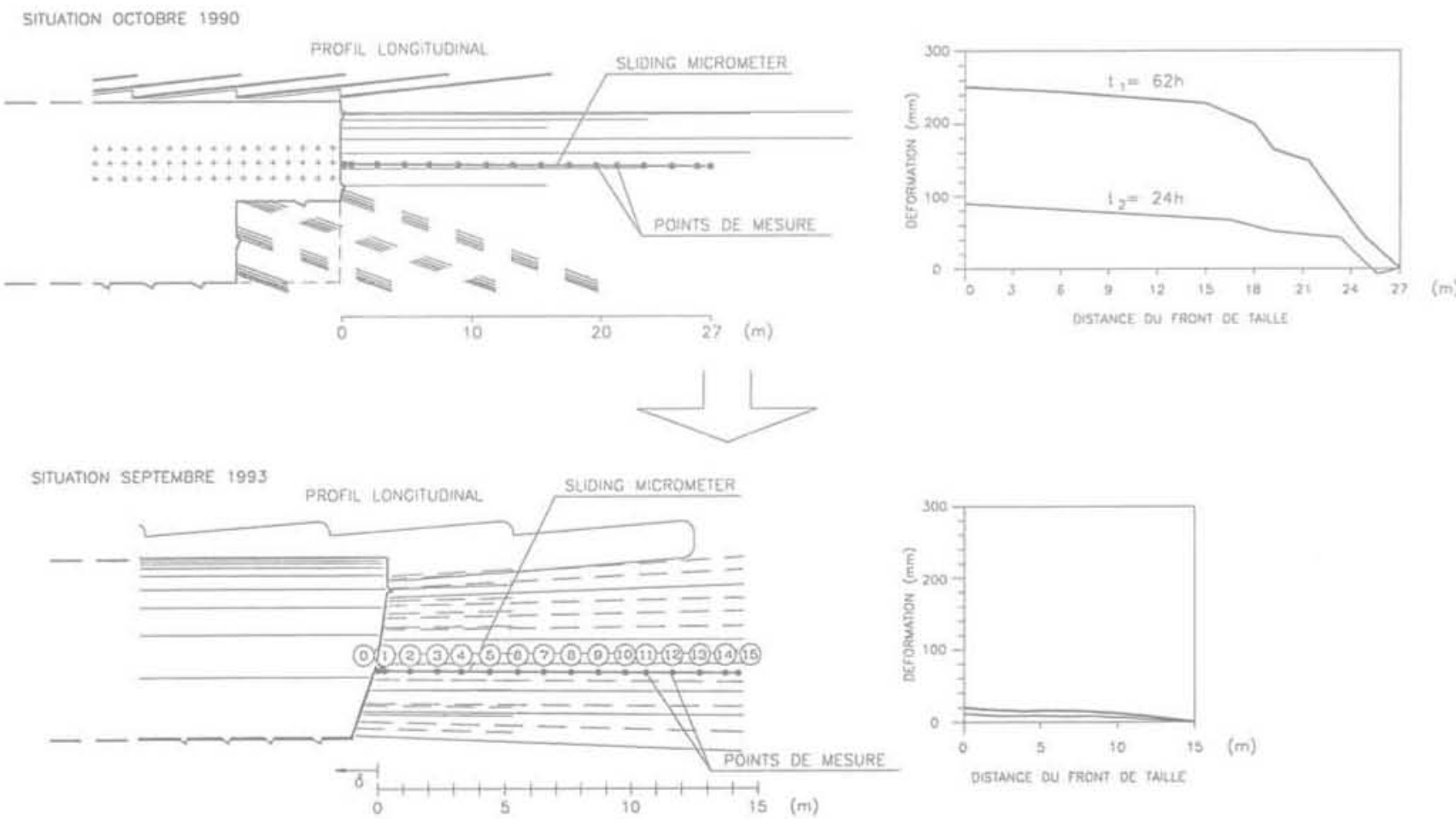

FG. 17 Ligne ferroviaire Caserta-Foggia - Tunnel de San Vitale : mesures d'extrusion au front de taille (phase de vérification).

Caserta-Foggia railway line - San Vitale tunnel: extrusion measurements of tunnel face (monitoring phase). 


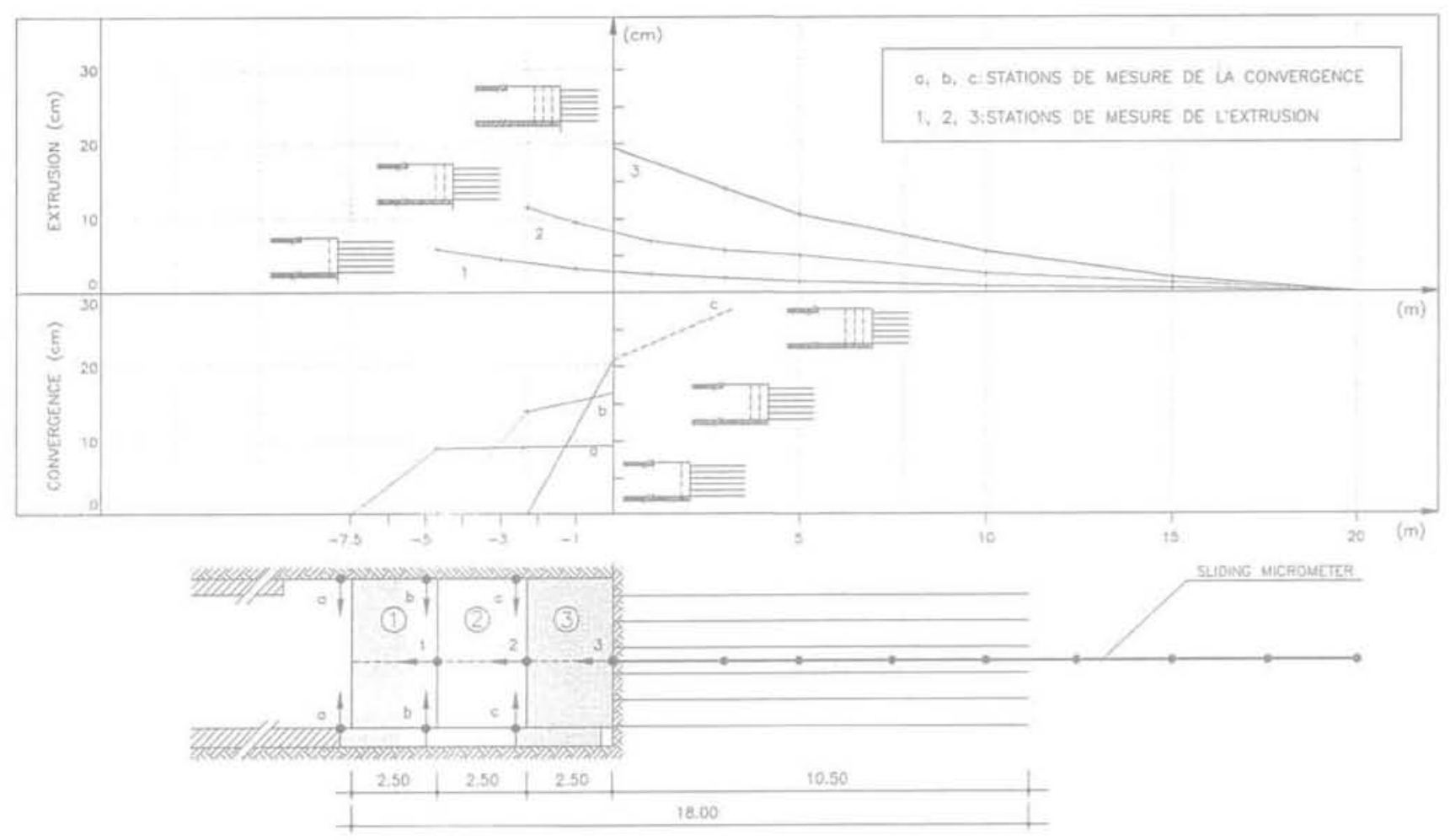

FG. 18 Ligne ferroviaire Caserta-Foggia - Tunnel de San Vitale; diagrammes extrusion-convergence en fonction de I'avancement (phase de vérification).

Caserta-Foggia railway line - San Vitale tunnel : extrusion-convergence diagrams as a function of the production (monitoring phase).

(convergences). Elles ont également révélé que les phénomènes de déformation de la cavité peuvent ètre contrôlés et sensiblement réduit en réglant artificiellement la déformabilité du noyau d'avancement et donc sa rigidité (confinement des extrusions). Ceci est possible grâce à l'exécution d'interventions appropriées de stabilisation dimensionnées et distribuées entre le noyau au front et la cavité, en fonction de la résistance et de la déformabilité du milieu ainsi que de la situation de contrainte existante.

Compte tenu de cela, dans le cas d'un milieu contraint en domaine élastoplastique :

- si l'état de contrainte en fonction des caractéristiques du milieu n'est pas trop élevé, il se peut qu'il suffise d'agir exclusivement sur la cavité par des interventions radiales, évitant toute intervention longitudinale sur le noyau d'avancement:

- si l'état de contrainte est élevé, il faudra en revanche agir surtout sur le noyau en le consolidant par des interventions longitudinales, évitant totalement les interventions radiales en aval du front de taille.

Au cas où le milieu serait contraint dans le domaine de rupture, il devient impératif de durcir le noyau d'avancement par des actions de préconfinement de la cavité qui pourront être intégrées à des actions de confinement appropriées en aval du front de taille. A cet égard, les expériences faites (et celles décrites précédemment sont particulièrement significatives) nous recommandent de travailler à l'avancement sur la forme et sur le volume du noyau au moyen de la réalisation d'une couronne de protection de terrain ren- forcé sur le contour du noyau. Au cours de la réalisation du tunnel de San Vitale, nous avons vu que pour surmonter les problèmes dans des sections particulièrement difficiles, il a été extrèmement utile d'opérer ainsi.

Si cela devait s'avérer insuffisant, il faudrait procéder à des interventions radiales supplémentaires de renforcement sur le contour de la cavité, dimensionnées pour absorber les convergences résiduelles que le noyau, bien que durci, n'est pas en mesure d'empêcher à lui seul.

\section{3}

\section{Le noyau d'avancement en tant qu'instrument de stabilisation}

Les résultats consécutifs à la recherche peuvent se résumer comme suit:

- dans la première phase de la recherche, nous avons défini trois typologies fondamentales de déformation (extrusion du front de taille, préconvergence et convergence de la cavité) et les manifestations d'instabilité qui en découlent (chutes de blocs sous l'effet du poids propre, écaillages, effondrement du front et écrasement de la cavité);

- dans la deuxième phase de la recherche, nous avons obtenu la confirmation expérimentale que tous les phénomènes de déformation (extrusion du front de taille, préconvergence et convergence de la cavité) 
ainsi que les manifestations d'instabilité visibles de l'intérieur de la cavité qui en découlent (chutes de blocs sous l'effet du poids propre, écaillages, effondrement du front et écrasement de la cavité) dépendent directement ou indirectement de la rigidité du noyau d'avancement:

- dans la troisième phase de la recherche, nous avons expérimentê qu'il était possible d'utiliser le noyau au front comme un instrument de stabilisation, en agissant artificiellement sur la rigidité du noyau pour contrôler les phénomènes de déformation de la cavité.

Par ailleurs, les résultats de la recherche:

- confirment notre opinion que la réponse en déformation du milieu au cours de l'excavation doit servir de point de référence principal au projeteur de tunnels, à plus forte raison parce qu'elle est l'indice de la formation et de la position de l'effet de voúte par rapport au profil d'excavation, autrement dit de la condition de stabilité atteinte par le tunnel:

- soulignent l'importance de tenir sous contrôle la réponse en déformation du système front de taillenoyau d'avancement au lieu de se limiter au seul contrôle de la cavité, dans la mesure où, comme nous l'avons vu, l'importance des charges agissant à long terme sur le revêtement dépend également de la rigidité du noyau:

- montrent que la réponse en déformation s'amorce en amont du front de taille, à la hauteur du noyau d'avancement et qu'elle évolue en aval, le long de la cavité; - indiquent clairement l'existence d'un lien direct entre la réponse en déformation du système front de taillenoyau d'avancement et celle de la cavité, dans le sens que cette dernière est la conséquence directe de la première;

- prouvent qu'en agissant sur la rigidité du noyau d'avancement par des interventions de protection et de renforcement, il est possible de contrôler sa déformabilité (extrusion, préconvergence) et par voie de conséquence, de contrôler également la réponse en déformation de la cavité (convergence).

En définitive, les résultats de la recherche permettent de considérer le noyau d'avancement comme un nouvel instrument de stabilisation à court et à long terme de la cavité. Un instrument dont la résistance et la déformabilité jouent un rôle déterminant dans la mesure où elles peuvent conditionner l'aspect qui doit préoccuper le plus le projeteur de tunnels, c'est-à-dire le comportement de la cavité à l'arrivée du front de taille.

C'est pourquoi, il est permis d'affirmer que le projeteur de tunnels doit, pour être à même de mettre en place un projet capable de garantir la stabilité à court et à long terme de l'ouvrage, concentrer toute son attention sur les phénomènes de contrainte et de déformation du système front de taille-noyau d'avancement, c'est-à-dire sur ses conditions de stabilité.

Il s'ensuit que le comportement du système front de taille-noyau d'avancement peut être adopté comme point de référence pour une standardisation des tunnels, avec en plus, l'avantage de constituer un paramètre conservant sa propre valeur quel que soit le type de terrain et quelle que soit la situation statique.

Dans cette optique, les trois situations fondamentales de contrainte et de déformation du système front de taille-noyau d'avancement définissent également les trois types possibles de comportement de la cavité (Fig. 19):

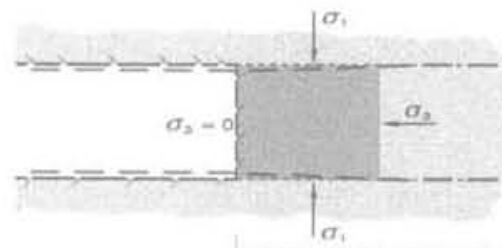

FRONT STABLE PRECONVERGENCE DE LA CAVITE.

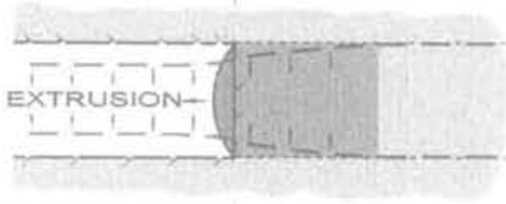

CONVERGENCE DE L CAVITE

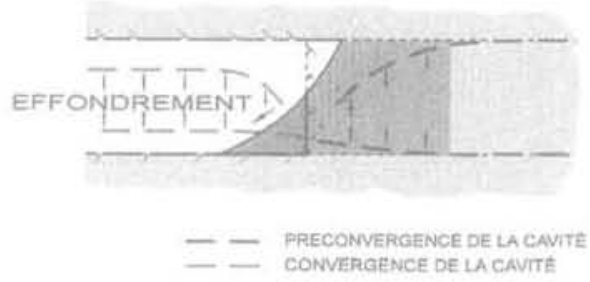

FRONT INSTABLE

FG. 19 Définition des catégories de comportement en se référant au noyau du front de taille considéré comme un instrument de stabilisation.

Definition of the behaviour categories, the nuclear of the tunnel face being considered as a stabilisation tool.

- comportement à front stable (catégorie de comportement A);

- comportement à front stable à court terme (catégorie de comportement B):

- comportement à front instable (catégorie de comportement C).

Dans la situation à front stable, la stabilité globale du tunnel est pratiquement assurée, même en l'absence d'interventions de stabilisation. Dans les situations B et $\mathrm{C}$, les résultats de la recherche indiquent que pour éviter les phénomènes d'instabilité du front et donc de la cavité, et pour essayer de revenir à une condition à front stable (A), il faut opérer par des interventions correctement équilibrées entre le front et la cavité et avec une intensité appropriée à la situation de contrainte réelle en fonction des caractéristiques de résistance et de déformabilité du milieu.

Si le système front de taille-noyau d'avancement constitue d'une part le "voyant lumineux» du comportement futur de la cavité et de l'autre, un instrument de stabilisation dans les mains du projeteur, if est nécessaire de pousser aux conséquences extrêmes les connaissances extraordinaires acquises et de développer, au niveau du projet et au niveau de la construction, une approche collant davantage à la réalité par rapport à celles qui ont été utilisées jusqu'ici.

L'approche selon l'analyse des déformations contrôlées dans les roches et dans les sols, dont les concepts et les informations seront illustrés dans une troisième étude, représente en ce sens la réponse logique et sans équivoque aux résultats de la recherche. 
[1] Kastner H. - Statik des Tunnel und Stollenbauses, 1962.

[2] Rabcewicz L.V. - «The New Austrian Tunnelling Methodn. Water Power 1969

[3] Tamez E. - « Estabilidad de tuneles excavados en suelos x. Conferenza presso la Mexican Engineering Academy, Mexico, 1984

[4] Kovari K. - "On the Existence of the NATM: Erroneus Concepts behind the New Austrian Tunnelling Method 1 . Tunnel, 1/1994.

[5] Lunardi P. - Progetto e costruzione di gallerie: presentazione dell'approccio ADECO-RS $x$. ISMES, Programma di istruzione permanente, Bergamo, 14-16 novembre 1988

[6] Lombardi G., Amberg W.A. - "Une méthode de calcul élasto-plastique de l'état de tension et de déformation autour d'une cavité souterraine $x$. International Congress ISRM, Denver, 1974
[7] Lunardi P., Bindi R., Focaraccia. «Nouvelles orientations pour le projet et la construction des tunnels dans des terrains meubles. Études et expériences sur le préconfinement de la cavité et la préconsolidation du noyau au front $\mathrm{x}$. Tunnels et micro-tunnels en terrain meuble, Paris 7-10 février 1989.

[8] Lunardi P. - "Aspetti progettuali e costruttivi nella realizzazione di gallerie in situazioni difficili: interventi di precontenimento del cavo x. Convegno Internazionale ell consolidamento del suolo e delle rocce nelle realizzazioni in sotterraneo v. Milan, 18-20 mars 1991.

[9] Lunardi P. Focaracci A., Giorgi P. Papacella A. - $\alpha$ Tunnel face reinforcement in soft ground design and controis during excavation 1 . International Congress Towards New Worlds in Tunnelling. Acapulco, 16-20 mai 1992.

[10] Lunardi P. - « Glass-resin tubes to stabilize the face of tunnels in difficult cohesive soils 1., SAIE, Seminario The application of Fiber Reinforced Plastics (FRP) in civil structural engineering, Bologna, 22 octobre 1993

[111 Lunardi P. - $\alpha$ La stabilité du front de taille dans les ouvrages souterrains en terrain meuble: études et expériences sur le renforcement du noyau d'avancement ». Symposium International Renforcement des sols : expérimentations en vraie grandeur des années quatre-vingt, Paris 18 novembre 1993. Presses des ponts et chaussées, 1993

[12] Lunardi P. - «Présoutènement et préconfinement $\mathrm{p}$. Revue française de géotechnique $n^{\circ} 80,1997$, p. 17-34.

[13] Autostrade S.p.A. - «Norme Tecniche d'Appalto m, 1992.

[14] Italferr Sis T.A.V. S.p.A - «Capitolato di Costruzione Opere Civilin, 1992

[15] A.N.A.S. - "Capitolato Speciale d'Appalto, Parte II: Norme Tecniche D, 1993 